\title{
Research on Loop Detection of Power Grid Equipment Based on Graph Database
}

\author{
Hongbin Qiu, Aihua Zhou, Kunlun Gao, Jiangpeng Dai, Bo Chai and Bo Zhang \\ Advanced Computing and Big Data Laboratory, Global Energy Interconnection Research Institute, State Grid Corporation of \\ China, Beijing, 102209, China
}

\begin{abstract}
-this paper presents a method of power grid loop detection based on graph database. The topology concept is introduced to extract the network relationship from the power grid equipment. The devices and power lines in the power grid correspond to the vertices in the topological graph, and the connection relation between the grid devices corresponds to the edges in the topological graph. The topological graph data model is generated by establishing the edges of connected vertices and stored in graph database. The loop detection of power grid equipment is divided into ordinary topological loop detection and multi-power loop detection, using the degree reduce algorithm and union-find sets to achieve the ordinary loop detection, and using the breadth first search algorithm to achieve the multipower loop detection. For large-scale power grid, the efficiency of power grid loop detection based on graph database can be 10 times higher than relational database.
\end{abstract}

Keywords-topological graph; graph database; loop detection; power grid

\section{INTRODUCTION}

In the power grid information system, the network topology characteristics of the power grid network is complicated. Power grid physical relationship, customer relationship, relationship of assets make network topology very huge, and produce a great amount of power grid operation data, monitoring data and meter data. At present, the network topology analysis system still use the traditional relational database to process massive data and analyze topology. The query efficiency, storage size, scalability and massive data management and many other aspects have encountered serious technical bottlenecks.

Graph database technology has great technological advantages in the application of network data structure, which can greatly improve the data query speed and computing performance. Graph data management technology made a major breakthrough in graph traversal, parallel processing and message transfer mechanism technology. It has been widely used in the Internet, social networking, e-commerce, bio genetic graph, intelligent transportation and other technical fields [1-2]. As the data structure of the graph has the technical advantage of the network characteristics of the power grid system, its application in the power grid calculation is gaining more attention. With graph data management technology and calculation model, data storage and numerical calculation can be easily combined. And distributed memory computing, parallel computing, decomposition aggregation and other core technologies can be made full use of, to meet the network topology analysis of massive data processing of large scale, high speed, high efficiency performance requirements. The power grid data management can be effectively supported. Billion scale multi voltage level power grid data analysis, including connected equipment analysis, charged state detection equipment and the power supply voltage across the query source analysis can be easily solved. The query time can be shorten, and provides a great potential solutions for the calculation of power grid data management and topology analysis system [3].

In view of the application on power grid system asset management and data query. It is of great technological advantage to use graph database to support topology analysis and optimization of power grid. Graph database using stored nodes and edges, with attribute record network topology information. It can improve the efficiency of power network topology analysis and storage characteristics at the same time. Meanwhile, graph database has real-time dynamic update, high query speed, and rich search function, can further improve the performance of power grid asset management and data query [4-6].

\section{Data StRacture OF POWER GRID EQUiPMENT}

The power grid devices and power lines correspond to the vertices in the graph. The connections among the power grid equipment, devices, and power lines correspond to the edges in the graph. And the graph data model is created by establishing the edges with connected vertices. Power grid topology and graph structure have a natural relationship that power grid equipment, power lines and their connections correspond to the vertices and edges of the graph structure. The graph model is constructed by the network topology, the connection between the power grid equipment is abstracted into vertex and edge connected based on graph database.

The attributes of the vertex of the graph correspond to the data structures picked up in the power grid geographic information system. Analyze the data structure of the vertex required, including device identification, device type, voltage level, switch status, switch, transformer, device port number, etc. According to the above data structure, the vertices and the edges of the vertex connection are established, and finally the topological graph is generated.

The power flow is transmitted along the edges, which is retrieved from the network topology source data, stored and recorded by the graph data, and the topological connection relation of the equipment and the circuit and the parameters of the equipment and the line are recorded. The graph data 
recording equipment - power line connection topology and equipment, line parameters, including power from power transmission and substation distribution of low voltage meter, higher flow line power topology, switch and branch line / transformer / equipment and tower line affiliation the asset management information system, establish records network topology and geographic information system information. Search in graph database, topology analysis, network traversal and path search based on established edges. According to the voltage level power grid topology analysis will be divided into different sub classification and analysis; network traversal need to use graph traversal technology, status updates for other equipment in the affected area; the path search is mainly used for the analysis of equipment connectivity, need to use the shortest path algorithm to achieve connectivity graph search query; analysis, establish the message through the iterative transfer model, the message passed the subordinate equipment vertex.

For power grid geographic information system topological relation management and query analysis, a power grid geographic information system analysis application system based on graph database is constructed. First of all, the service data of power grid geographic information system to sort out the merger of uniform dispersed in the device data in each table field, and according to the characteristics of data storage are summarized, for the formation of calculation analysis and application of the data storage structure of power network topology architecture of geographic information system. Then, the topology of power grid geographic information system analysis functions are classified into different business logic, including the following three categories: network traversal: charged power point analysis, initialization, set the range of power failure analysis, switch state change charged analysis, topology search: search, the topological query, path searching, two equipment connectivity analysis.

With the transformation of the power grid topology, the online updating of the graph database always ensures the corresponding relationship between the two. When the network topology of electrical components and the connecting lines add, removal, change, real-time batch update graph database of the vertices and edges of the corresponding batch add, change, remove, always keep the actual power topology and graph database consistency.

\section{LOOP DETECTION PROCESS MODEL}

The power grid equipment information is expressed according to graph theory in the form of vertices, edges and attributes. The power grid equipment represented as vertices, the connection between the equipment for equipment information grid edge, expressed as vertex attributes, connection information represented as edge attributes, and the construction of power network topology according to the relationship between vertex and edge, the formation of power grid equipment undirected topology. The loop detection of power grid equipment is divided into ordinary topological loop detection and multi-power loop detection. The flow chat of loop detection based on graph database is shown in figure 1.

\section{1) Ordinary topology loop detection}

By finding the voltage level field in the vertex attribute, the vertices of the same voltage level as the starting point are filtered, and the vertices of each vertex are obtained by counting the number of connected edges of each vertex. Delete the vertices in the topology of 1 and all their join edges, then delete the operations in the newly generated graph until the vertices are not 1 , and record the final topology. If the final vertex is not included in the final graph, the normal topology loop is not detected from the starting vertex; if the final graph contains the starting vertex, the Unicom subgraph with the starting vertex is found. By using the algorithm, the number of ordinary topological loop paths detected from the beginning vertices is obtained, and the single ring paths of each normal topological loop are recorded.

\section{2) Multi-power loop detection}

Starting from the starting point, through the breadth first search, traversing all the reachable vertices of the same voltage level, the device type is found to be the vertex of the highvoltage transformer. If you don't find a step-down transformer over 1 high voltage level, from the initial vertex of detected multi loop power supply; if found more than 1 step-down transformer high voltage level, are recorded from the initial vertex to vertex of each path topology step-down transformer high voltage. When the number of high voltage class step-down transformers is $n$, the number of multi-power loop paths is (n-1) $\mathrm{n} / 2$. A single loop path of each of the multi-power supply loops is obtained by merging the topological paths of the step-down transformer vertices from the starting point to the high voltage level.

After the total number of network loops is counted and the single loop path of each loop is recorded, the total number of power grid loops is the sum of the number of ordinary topological loop paths and the number of paths of multi-power loops. 


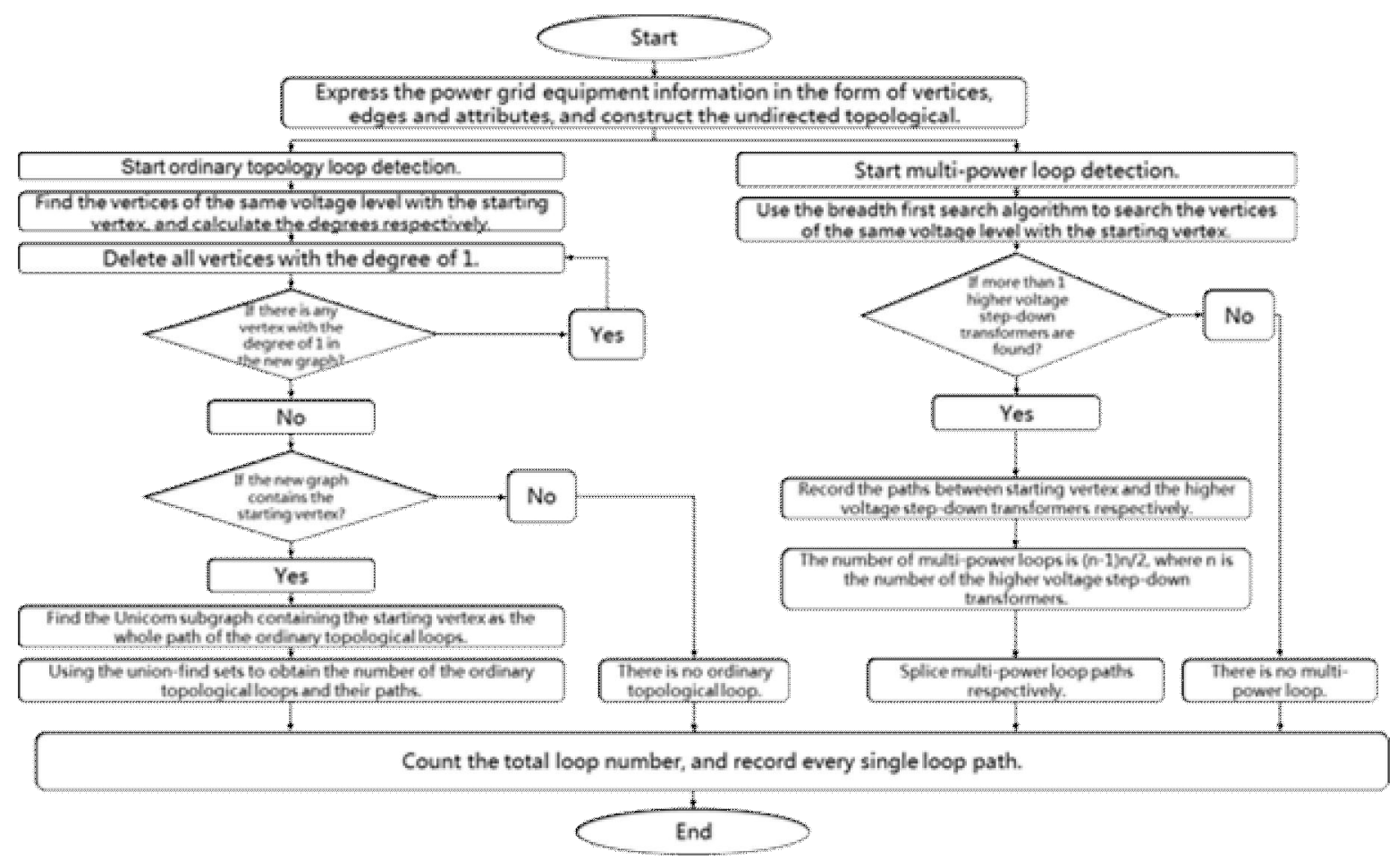

FIGURE I. FLOW CHAT OF LOOP DETECTION

\section{EXPERIMENT RESULTS}

A comparative test based on power grid equipment data of Shanghai was made. This data contains 31 million vertices and 78 million edges. The performance test of power grid equipment is carried out respectively by using the method of power grid loop detection based on graph database and power grid loop detection method based on relational database. Performance tests in scenarios with and without loops.

In the scenario with loops, there are 3 ordinary loops and 2 multi-power loops, a total of 5 loops. The running time results of the two kinds of database are shown in table 1.

\section{TABLE I. RUN TIME CONTRAST IN THE SCENARIO WITH LOOPS}

\begin{tabular}{|l|c|c|c|}
\hline \multirow{2}{*}{$\begin{array}{c}\text { Total of 5 } \\
\text { Loops }\end{array}$} & \multicolumn{3}{|c|}{ Run Time } \\
\cline { 2 - 4 } & Average & $\mathbf{9 0 \%}$ Line & Max \\
\hline $\begin{array}{l}\text { Graph } \\
\text { Database }\end{array}$ & 0.706 & 0.815 & 1.292 \\
\hline $\begin{array}{l}\text { Relational } \\
\text { Database }\end{array}$ & 7.078 & 8.037 & 10.833 \\
\hline
\end{tabular}

In the scenario without loop, the running time results of the two kinds of database are shown in table 2.

TABLE II. RUN TIME CONTRAST IN THE SCENARIO WITHOUT LOOP

\begin{tabular}{|l|c|c|c|}
\hline \multicolumn{1}{|c|}{$\begin{array}{c}\text { 0 Loop } \\
\text { Path }\end{array}$} & \multicolumn{3}{|c|}{ run time } \\
\cline { 2 - 4 } & Average & 90\% Line & Max \\
\hline $\begin{array}{l}\text { Graph } \\
\text { Database }\end{array}$ & 0.576 & 0.64 & 1.295 \\
\hline $\begin{array}{l}\text { Relational } \\
\text { Database }\end{array}$ & 6.803 & 7.765 & 11.324 \\
\hline
\end{tabular}

Through the experimental results, it can be seen that the two methods can correctly accomplish the loop detection task. The difference of running time with and without loops is not obvious. The running time of power grid loop detection method based on graph database is $1 / 10$ of the running time based on the relational database. The efficiency of the method based on graph database is 10 times higher.

\section{CONCLUSIONS}

This paper proposes a method of power grid loop detection based on graph database to solve the problem of power grid equipment topology analysis, from the analysis of the test results can be seen, the power grid graph database loop detection method can quickly and effectively provide the loop topology detection based on loop detection, improve efficiency. As an important topological analysis function of power grid topology analysis system based on graph database, the detection of power grid loop is an indispensable function module in power grid topology analysis. This method will lay the foundation for upgrading conditions under the platform of power grid geographic information system generation technology, solve the technical difficulties of topology and performance bottlenecks in the service, to further promote the power of lean management and global energy network construction.

\section{REFERENCES}

[1] Malewicz G, Austern M H, Bik A J C, et al. Pregel: a system for largescale graph processing[C]// SPAA 2009: Proceedings of the 21st Annual ACM Symposium on Parallelism in Algorithms and Architectures, Calgary, Alberta, Canada, August 11-13, 2009. 2009:135--146. 
[2] Vicknair C, Macias M, Zhao Z, et al. A comparison of a graph database and a relational database: a data provenance perspective $[\mathrm{C}] / /$ Proceedings of the 48th annual Southeast regional conference. ACM, 2010: 42.

[3] Angles R. A comparison of current graph database models[C]//Data Engineering Workshops (ICDEW), 2012 IEEE 28th International Conference on. IEEE, 2012: 171-177.

[4] He H, Singh A K. Closure-tree : An index structure for graph queries $[\mathrm{C}]$. ICDE2006 (Proceedings of the 22nd International Conference on IEEE 2006）， $2006: 38 \sim 38$

[5] Gonzalez J E, Xin R S, Dave A, et al. Graphx:Graph processing in a distributed dataflow framework. Proceedings of the 11th USENIX Symposium on Operating Systems Design and Implementation . 2014.

[6] Semih Salihoglu, Jennifer Widom. GPS: A Graph Processing System[C] 25th International Conference on Scientific and Statistical Database Management, SSDBM 2013, 2013: 1-31. 